\title{
Intensity-dependent relaxation of photoconductivity in nanocrystalline titania thin films
}

\author{
Z. Xie, ${ }^{1}$ V. M. Burlakov, ${ }^{1, *}$ B. M. Henry, ${ }^{1}$ K. R. Kirov,${ }^{1}$ H. E. Smith, ${ }^{1}$ C. R. M. Grovenor, ${ }^{1}$ H. E. Assender, ${ }^{1}$ G. A. D. Briggs, \\ Mitsuru Kano, ${ }^{2}$ and Yusuke Tsukahara ${ }^{2}$ \\ ${ }^{1}$ Department of Materials, University of Oxford, Parks Road, Oxford OX1 3PH, United Kingdom \\ ${ }^{2}$ Technical Research Institute, Toppan Printing Co., Ltd., Takanodai-Minami 4-2-3, Sugito-machi, Kitakatsushika-gun, \\ Saitama 345, Japan \\ (Received 6 October 2005; revised manuscript received 27 February 2006; published 31 March 2006)
}

\begin{abstract}
We have discovered that the initial rate of the post-illumination decay of photoconductivity in nanocrystalline $\mathrm{TiO}_{2}$ depends on the intensity of the illumination. The phenomenon is described by hole detrapping processes affected by electrostatic interactions between the trapped holes, and screening of these interactions by free electrons. The analysis allows determination of both the electron concentration and the electron mobility. In our materials, the value of electron mobility $\mu \approx 10^{-6} \mathrm{~cm}^{2} \mathrm{~V}^{-1} \mathrm{~s}^{-1}$, in good agreement with results obtained by other methods.
\end{abstract}

DOI: 10.1103/PhysRevB.73.113317

The wide-band-gap semiconductor $\mathrm{TiO}_{2}$ is often used as an electron acceptor in composite solar cells (CSC's) consisting of conjugated polymer acting both as a light absorber and as a hole conductor. Excitons photogenerated in the conjugated polymer can dissociate at the polymer/titania interfaces injecting electrons into the $\mathrm{TiO}_{2}{ }^{1}$ Hence high quantum efficiency in a CSC requires a large organic-inorganic interface area, which in turn suggests a need for a high porosity in the titania. In addition to the exciton and charge transport properties of the polymer, CSC efficiency strongly depends on the efficiency with which the injected electrons can pass through the porous nanocrystalline titania film to reach the anode. Electron transport is heavily affected by both the crystalline imperfections in the $\mathrm{TiO}_{2}$ nanocrystals ${ }^{2,3}$ and the film morphology, ${ }^{4,5}$ such as effective surface area. Techniques used to explore the transport properties include time of flight, ${ }^{6}$ photocurrent transients, ${ }^{7}$ spectroelectrochemical method, ${ }^{8}$ and intensity-modulated photocurrent spectroscopy. ${ }^{9}$ The main parameters of interest are electron mobility and diffusion coefficient. Assuming the concentration of free electrons to be in the range of $10^{18}-10^{19} \mathrm{~cm}^{-3}$, the electron drift mobility is found to be in the range of $10^{-7}-10^{-4} \mathrm{~cm}^{2} \mathrm{~V}^{-1} \mathrm{~s}^{-1}$.

In this paper, we report a phenomenon of the dependence of post-illumination photoconductivity relaxation in porous $\mathrm{TiO}_{2}$ films upon illumination intensity. Analysis of this phenomenon in terms of electron transport affected by recombination of free electrons with detrapped holes, and taking into account that hole detrapping is influenced by screened electrostatic interactions, explains both the intensity and temperature dependence of the photoconductivity relaxation, and allows simultaneous determination of the electron concentration and mobility.

Photoconductivity (PC) measurements on nanocrystalline titania thin films fabricated by a sol gel method ${ }^{10,11}$ were performed in vacuum. As anatase is very sensitive to the presence of oxygen and water, ${ }^{12,13}$ the samples were annealed at $380 \mathrm{~K}$ in vacuum for $2 \mathrm{~h}$ to remove traces of absorbed water prior to each PC measurement. Annealing did not affect the sample microstructure, as no change in conductivity after annealing has been detected. Current-voltage $(I-V)$ characteristics measured in the dark show diodelike
PACS number(s): 78.66.Li, 73.63.Bd, 73.50.Gr, 73.50.Pz

behavior due to the rectifying properties of the $\mathrm{TiO}_{2} / \mathrm{Au}$ interface and low concentration of charge carriers. But linear $I-V$ characteristics are observed during, and for a substantial period after, illumination at all intensities used in this study. All PC measurements were performed in the linear regime under low bias $(0.5 \mathrm{~V})$, so that the conductivity is dominated by photogenerated carrier density. Since the titania is quite conductive under illumination, the total resistance is close to that of the ITO electrode. Conductivity of these titania films was calculated as $\sigma=d S^{-1}\left(V / I-R_{\text {IтO }}\right)$, taking into account the ITO electrode resistance, where $d=150 \mathrm{~nm}$ is titania film thickness, $S=0.063 \mathrm{~cm}^{2}$ is the tested area, $V=0.5 \mathrm{~V}$ is the applied voltage, $I$ is the measured current $(A)$, and $R_{\mathrm{ITO}}=150 \Omega$ is the ITO electrode resistance. The latter was measured for control samples without titania layers. The measured dark conductivity at room temperature, $1.8 \times 10^{-11} \Omega^{-1} \mathrm{~m}^{-1}$, is in good agreement with values reported for similar materials. ${ }^{14}$

We found that the $\mathrm{PC}$ in nanocrystalline $\mathrm{TiO}_{2}$ steadily increases with illumination time, and decreases remarkably slowly after the illumination is switched off (see Fig. 1). Similarly slow PC decay rates have been observed in $\mathrm{Nb}$ doped $\mathrm{TiO}_{2},{ }^{15} \mathrm{ZnO},{ }^{16}$ and $\mathrm{TiO}_{2}$ (Refs. 17-19) thin nanocrystalline films, with relaxation times ranging from $10^{-2}$ to more than $10^{4} \mathrm{~s}$. This very slow relaxation of PC was attributed to carrier detrapping effects and slow recombination of electrons and holes at grain boundaries in the nanocrystals. ${ }^{15,16} \mathrm{~A}$ new effect was discovered when we carefully analyzed the post-illumination relaxation of PC. As can be seen from Fig. 2, the initial rate of decrease of normalized PC is slower if the sample was previously illuminated with a higher light intensity. Such an effect cannot be related to the sample temperature variation under different illumination intensity. As we show in this paper, an increase of sample temperature would result in a quicker PC decay, which is in contrast to experimental findings (see Fig. 3 and Ref. 19).

The time dependence of PC has been previously modeled in nanocrystalline $\mathrm{TiO}_{2}$ using a broad spectrum of charge carrier traps. ${ }^{20}$ The processes of trap filling and emptying, competing with recombination, can fairly simply explain the observed slow rise and decay of PC. The higher the light intensity, the quicker the traps are filled and PC saturation is 


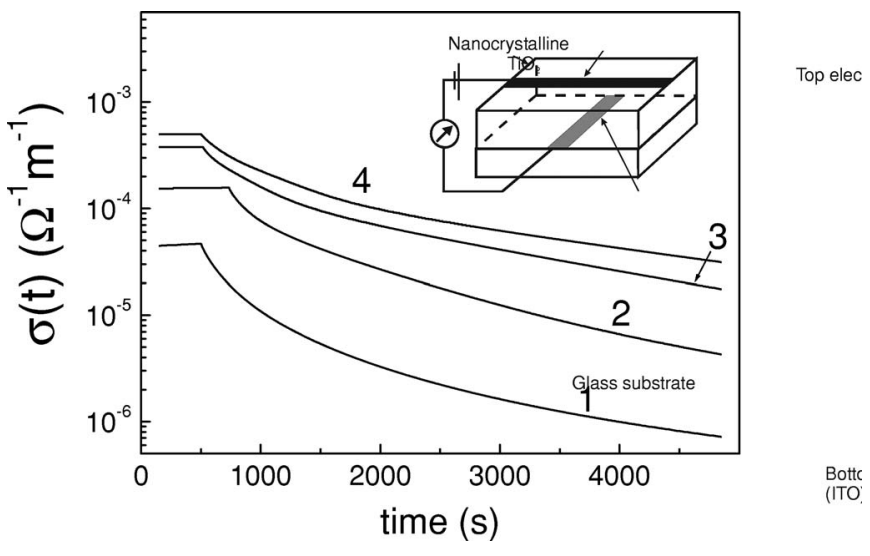

FIG. 1. Post-illumination decay of conductivity measured in nanocrystalline $\mathrm{TiO}_{2}$. Curves correspond to illumination light intensities of (1) 0.5 , (2) 1 , (3) 3 , and (4) $10 \mathrm{~mW} / \mathrm{cm}^{2}$. The kinks in the curves correspond to the times when the illumination is switched off. The inset shows the experimental setup for the conductivity measurements.

achieved. It is more difficult to explain the intensity dependence of the PC decay. We shall describe a model that takes into account hole trapping and electron screening of electrostatic interactions between trapped holes. It is worth pointing out that the mean-field analysis described below does not imply any particular mechanism of carrier trapping/ detrapping.

Consider an intrinsic semiconductor with microstructurally induced random fluctuations of local electronic energy. These fluctuations in energy can be associated with fluctuations of electrostatic potential about an average level, which we set to zero. The negative fluctuations of the potential can be associated with negatively charged traps for holes. According to the charge neutrality of the intrinsic semiconductor, an average charge density associated with these traps is totally compensated by that associated with positive fluctuations of the potential. The electron mobility in $\mathrm{TiO}_{2}$ is four orders of magnitude higher than the hole mobility. ${ }^{8}$ This suggests that localization of electrons on positive fluctuations of the potential is much weaker than localization of holes on negative fluctuations of the potential, so that holes spend much longer time on localization centers than electrons do. The number of localized electrons is therefore expected to be much lower than that of localized holes. We consider for

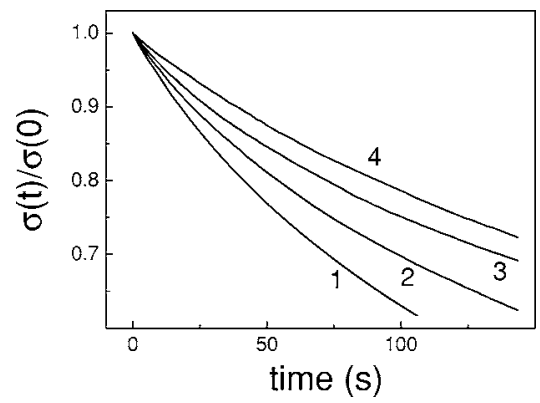

FIG. 2. Normalized conductivity as a function of time after switching off illumination light of intensity: (1) 0.5 , (2) 1.0, (3) 3.0, and (4) $10.0 \mathrm{~mW} / \mathrm{cm}^{2}$.

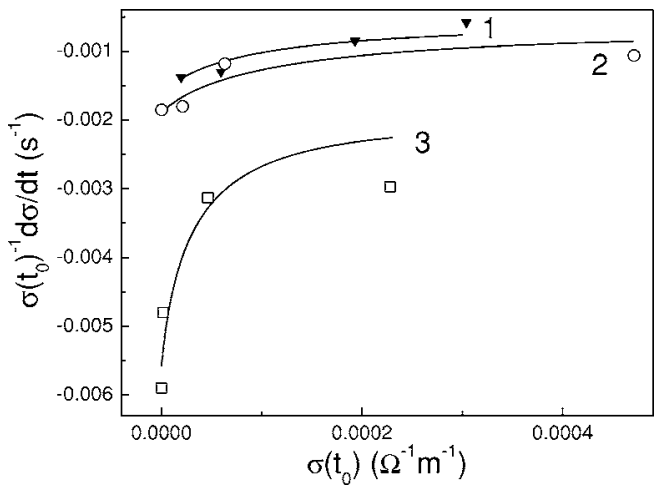

FIG. 3. Relative initial decay rate of PC as a function of conductivity $\sigma\left(t_{0}\right)$ (hence illumination intensity) for two different samples of nanocrystalline $\mathrm{TiO}_{2}$ at $T=295 \mathrm{~K}$ after switching off the illumination. Symbols mark experimental values obtained at illumination levels shown in Figs. 1 and 2, and the curves are model fits with the function given by Eq. (9). Solid triangles with curve (1) and open circles with curve (2) correspond to two different pixels of sample 1; open squares and curve (3) correspond to sample 2. The fit parameters values are (1) $C=0.00068, P=160$, (2) $C=0.00071, P=138$, (3) $C=0.00213, P=260$. Corresponding values for electron mobility are $\mu \simeq 1.9 \times 10^{-6} \mathrm{~cm}^{2} \mathrm{~V}^{-1} \mathrm{~s}^{-1}$ (1), $2.5 \times 10^{-6} \mathrm{~cm}^{2} \mathrm{~V}^{-1} \mathrm{~s}^{-1}(2), 0.7 \times 10^{-6} \mathrm{~cm}^{2} \mathrm{~V}^{-1} \mathrm{~s}^{-1}$ (3).

simplicity the extreme case when all photo-generated holes are localized (it is implicit that the concentration of holes is much lower than that of hole traps suggesting the illumination intensity to be sufficiently low), and all electrons are free. ${ }^{21}$ Even if a fraction of the electrons are localized, their residence time on the trap levels would be much shorter than that for holes, and will not noticeably affect the results of our model.

We can assume from the low value of conductivity of nanocrystalline $\mathrm{TiO}_{2}$ in the dark relative to that under illumination that the concentration of electrons injected into $\mathrm{TiO}_{2}$ from the cathode is negligible compared to the number of photogenerated electrons. Therefore, one may conclude that the concentration $n(t)$ of free electrons in the system is controlled by the concentration of trapped holes $h(t)$, and the conductivity $\sigma(t)$ can be expressed as

$$
\sigma(t)=e \mu n(t)=e \mu \int_{E_{\min }}^{E_{\max }} h(E, t) g(E) d E,
$$

where $h(E, t)$ is the probability of a trap with energy level $E$ being occupied at a time $t$ by a hole, $g(E)$ is the trap density of states, $E$ is the trap energy measured from the hole mobility edge, and $\mu$ is the electron mobility, which is assumed to be a constant.

In the absence of the photogenerated carriers, the average electrostatic energy of the system is set to zero by definition. As the photogenerated free carriers of both signs are evenly distributed in space, they produce zero total charge density and hence an electrostatic energy of their interaction is also zero. Localization of a photogenerated hole on a negatively charged trap compensates trap charge and results in an asymmetry between positive and negative charge fluctuations, thus increasing the electrostatic energy in the system..$^{22}$ This 
electrostatic energy is decreased due to screening by free electrons, and therefore depends on electron concentration. Thermal emission of a hole from the trap level would then depend on the concentration of holes, as concentration of trapped holes is assumed to be equal to concentration of free electrons. To keep the number of model parameters low, we make one more assumption, the implications of which we will discuss later. We assume that the rate-determining process for PC decay is the thermal emission of holes from traps (detrapping) rather than recombination of trapped holes with free electrons. Once a hole is detrapped, it can be assumed to immediately recombine, as trapping again of the detrapped hole formally results in an extension of holes residence time on a trap level, and can effectively be taken into account by renormalizing the trap energies.

Time evolution of the trap occupation number $h(E, t)$ is controlled by the trapped hole generation rate $G_{E}$ and the thermal ionization rate of traps at temperature $T$ with attempt frequency $f$. This can be expressed as

$$
\frac{d h(E, t)}{d t}=G_{E}-f h(E, t) \exp \left(-\frac{E-\Delta(h(t))}{k_{B} T}\right),
$$

where $h(t)=\int h(E, t) g(E) d E$ is hole concentration, and $\Delta(h(t))$ is an energy of electrostatic interaction between trapped holes, which reduces the trap ionization energy, and $k_{B}$ is the Boltzmann constant. Substituting Eq. (2) into the time derivative of Eq. (1), one obtains

$$
\begin{aligned}
\frac{d \sigma^{\mathrm{on}}(t)}{d t}= & e \mu \int_{E_{\min }}^{E_{\max }}\left[G_{E}-f h(E, t)\right. \\
& \left.\times \exp \left(-\frac{E-\Delta(h(t))}{k_{B} T}\right)\right] g(E) d E,
\end{aligned}
$$

where the superscript "on" refers to the process under illumination. In Eq. (3), we have neglected the dependence of $g(E)$ upon electrostatic interactions, assuming that at any time $\left|E_{\min }\right| \gg|\Delta(h(t))|$ since the main contribution is from deep traps.

In the simplest case, each trap has a single energy level, and $g(E)$ then represents the concentration of traps with energy $E$. If a probability for a trap to capture a hole does not depend on the trap energy, then $G_{E}$ can be expressed as $G_{E}=G / N_{t}, N_{t}=\int_{E_{\min }}^{E_{\max }} g(E) d E$, where $G$ is the overall hole generation rate, which is proportional to the incident light intensity, and $N_{t}$ is the trap concentration. Substituting $G_{E}$ into Eq. (3), we have

$$
\begin{aligned}
\frac{d \sigma^{\mathrm{on}}(t)}{d t}= & e \mu G-e \mu f \\
& \times \int_{E_{\min }}^{E_{\max }}\left[h(E, t) \exp \left(-\frac{E-\Delta(h(t))}{k_{B} T}\right)\right] g(E) d E .
\end{aligned}
$$

Equation (4) gives dependence of the PC growth rate on hole generation rate $G$ (and hence on light intensity) and on the sample temperature $T$ in the regime controlled by hole detrapping processes.
To model the relaxation of PC, it is assumed that the illumination is switched off at a time $t_{0}$. Hole occupation numbers at $t_{0}$ can then be obtained by solving the equation

$$
h\left(E, t_{0}\right)=\frac{1}{e \mu f N_{t}} \exp \left(\frac{E-\Delta\left(h\left(t_{0}\right)\right)}{k_{B} T}\right)\left[e \mu G-\left(\frac{d \sigma^{\mathrm{on}}(t)}{d t}\right)_{t_{0}}\right] \text {. }
$$

The initial $\left(t \approx t_{0}\right)$ relative rate of PC decay can be described by substituting Eq. (5) into Eq. (3) with $G_{E}=0$,

$$
\frac{1}{\sigma_{0}}\left(\frac{d \sigma^{\mathrm{off}}(t)}{d t}\right)_{t_{0}}=-C \exp \left(\frac{\Delta\left(h_{0}\right)}{k_{B} T}\right)
$$

where $\sigma_{0}=\sigma\left(t_{0}\right)$ and $h_{0}=h\left(t_{0}\right)$, and the constant $C=f N_{t} /\left[\int_{E_{\min }}^{E_{\max }} \exp \left(E / k_{B} T\right) g(E) d E\right]$ depends only on temperature. Equation (6) gives a qualitative description of the PC relative decay rate as a function of temperature. To illustrate this temperature dependence, assume a boxcar distribution of hole density of states, i.e., $g(E)=N_{t}\left(E_{\max }-E_{\min }\right)^{-1}$ if $E_{\max }>E>E_{\min }$, and $g(E)=0$ otherwise. Then assuming $E_{\max } \gg E_{\min } \gg|\Delta(h(t))|$ and $E_{\max } \gg k_{B} T$, Eq. (6) can then be reduced to

$$
\frac{1}{\sigma_{0}}\left(\frac{d \sigma^{\mathrm{off}}(t)}{d t}\right)_{t_{0}} \simeq-f\left(E_{\max }-E_{\min }\right) \frac{\exp \left(-E_{\max } / k_{B} T\right)}{k_{B} T}
$$

suggesting that photoconductivity relative decay rate is higher at higher sample temperature. This result is in qualitative agreement with experimental observations.

The average electrostatic interaction energy $\Delta\left(h_{0}\right)$ can be estimated assuming a continuous distribution of trapped holes in the bulk of $\mathrm{TiO}_{2}$ and neglecting the surface effects according to the arguments presented earlier,

$\Delta\left(h_{0}\right) \simeq \frac{h_{0} e^{2}}{\varepsilon \varepsilon_{0}} \int_{R_{0}}^{\infty} r \exp \left(-\frac{r}{r_{D}}\right) d r=k_{B} T\left(1+\frac{R_{0}}{r_{D}}\right) e^{-R_{0} r_{D}}$,

where $r_{D}=\sqrt{k_{B} T \varepsilon_{0} \varepsilon / h_{0} e^{2}}$ is the Debye screening radius. When estimating $\Delta\left(h_{0}\right)$ in Eq. (9), we took into account that the distance between two trapped holes is normally larger than some minimum distance $R_{0}$. The latter can be estimated as $R_{0} \approx N_{t}^{-1 / 3} \approx 5 \mathrm{~nm}$ taking it as an average distance between the traps at a concentration $N_{t} \approx 10^{19} \mathrm{~cm}^{-3} \cdot{ }^{12,18}$ Substituting Eq. (8) into Eq. (6) and taking into account that $h_{0}=\sigma_{0} /(e \mu)$, one obtains

$$
\begin{gathered}
\frac{1}{\sigma_{0}}\left(\frac{d \sigma^{\mathrm{off}}(t)}{d t}\right)_{t_{0}}=-C \exp \left[\left(1+P \sigma_{0}^{1 / 2}\right) \exp \left(-P \sigma_{0}^{1 / 2}\right)\right], \\
P=R_{0} \sqrt{\frac{e}{k_{B} T \mu \varepsilon_{0} \varepsilon}},
\end{gathered}
$$

where $\varepsilon_{0}$ and $\varepsilon$ are the dielectric constants of vacuum and of $\mathrm{TiO}_{2}$, respectively.

Equation (9) with two fitting parameters $C$ and $P$ was used to fit the experimental data. Initial relative decay rate of photoconductivity in two different samples of nanocrystalline $\mathrm{TiO}_{2}$ as a function of conductivity (hence light intensity) is shown in Fig. 3. Lines show the fit of experimental data 
with Eq. (9). Taking $\varepsilon \approx 20$ as the dielectric constant of the porous nanocrystalline titania [an average dielectric constant for the bulk anatase crystal is $\varepsilon \simeq 37$ (Ref. 23)], the roomtemperature electron mobility is found for different samples to be of the order of $\mu \approx 10^{-6} \mathrm{~cm}^{2} \mathrm{~V}^{-1} \mathrm{~s}^{-1}$ within the range of values reported by other groups. ${ }^{6-9}$ Using the value for electron mobility, one can estimate the electron (hole) concentration $n_{0}$ as a function of illumination conditions according to the relation $n_{0}=h_{0}=\sigma_{0} /(e \mu)$. To fulfill the model assumption about all holes being localized at the estimated trap concentration $10^{19} \mathrm{~cm}^{-3}$, the illumination level should be chosen low enough to avoid saturating all traps. Taking according to this requirement an illumination intensity $\sim 3 \mathrm{~mW} / \mathrm{cm}^{2}$, the electron concentration is found to be $(0.3-0.7) \times 10^{19} \mathrm{~cm}^{-3}$, i.e., of the order of the trap concentration $N_{t}$. At illumination levels higher than $10 \mathrm{~mW} / \mathrm{cm}^{2}$, total trap filling may result in an electron concentration exceeding $N_{t}$ due to the presence of photogenerated free holes, which is not accounted for in our model.

It is worth commenting on our central assumption about the rate-determining process of the PC decay being determined by hole detrapping rather than recombination of trapped holes with electrons. ${ }^{24}$ If a hole trap has roughly the same (but with opposite sign) charge as the hole, which we believe is the case for deep traps, then localization of the hole would result in the formation of an almost neutral complex. Electron scattering on such a complex, and conse- quently recombination with the localized hole, would then be suppressed. The contribution of recombination to the PC decay should increase with increasing concentration of the charge carriers and hence light intensity, and is therefore not supported by the experimentally observed trend.

In conclusion, we have discovered a phenomenon of photoconductivity relaxation with the relaxation rate dependent on the intensity of the previous illumination of nanocrystalline $\mathrm{TiO}_{2}$. The phenomenon is interpreted within the model based on the concept of hole detrapping affected by electrostatic interactions between trapped holes (or noncompensated charge fluctuations in the medium), and by electron screening of these interactions. From the model fit of experimental data, we have extracted an estimate for the value of electron mobility in nanocrystalline $\mathrm{TiO}_{2}$ of $\mu \approx 10^{-6} \mathrm{~cm}^{2} \mathrm{~V}^{-2} \mathrm{~s}^{-1}$ and carrier concentration $n_{0} \approx 10^{18}-10^{19} \mathrm{~cm}^{-3}$. The model accounts also for the temperature dependence of photoconductivity decay in $\mathrm{TiO}_{2}$, and is applicable to a wide range of strongly disordered semiconductors with one type of carrier localized.

We thank Professor G. D. W. Smith (FRS) for the provision of laboratory facilities. We are also grateful to Dr. M. J. Carey and R. Turner for their help in setting up the PC experiments, and to Dr. J. Nelson for useful discussions. The financial support of the Toppan Printing Company Ltd., Japan is gratefully acknowledged. G.A.D.B. thanks EPSRC for financial support.
*On leave from the Institute for Spectroscopy Russian Academy of Sciences, Troitsk 142190, Moscow region, Russia.

${ }^{1}$ C. D. Grant, A. M. Schwartzberg, G. P. Smestad, J. Kowalik, L. M. Tolbert, and J. Z. Zhang, Synth. Met. 132, 197 (2002).

${ }^{2}$ J. Nelson, S. A. Haque, D. R. Klug, and J. R. Durrant, Phys. Rev. B 63, 205321 (2001).

${ }^{3}$ G. Franco, J. Gehring, L. M. Peter, E. A. Ponomarev, and I. Uhlendorf, J. Phys. Chem. B 103, 692 (1999).

${ }^{4}$ A. C. Arango, L. R. Johnson, V. N. Bliznyuk, Z. Schlesinger, S. A. Carter, and H. Hörhold, Adv. Mater. (Weinheim, Ger.) 12, 1689 (2000).

${ }^{5}$ A. J. Frank, N. Kopidakis, and J. van de Lagemaat, Coord. Chem. Rev. 248, 1165 (2004).

${ }^{6}$ B. O. Aduda, P. Ravirajan, K. L. Choy, and J. Nelson, Int. J. Photoenergy 6, 141 (2004).

${ }^{7}$ A. Solbrand, H. Lindstrom, H. Rensmo, A. Hagfeldt, S. E. Lindquist, and S. Sodergren, J. Phys. Chem. B 101, 2514 (1997).

${ }^{8}$ L. Forro, O. Chauvet, D. Emin, L. Zuppiroli, H. Berger, and F. Levy, J. Appl. Phys. 75, 633 (1994).

${ }^{9}$ B. van der Zanden and A. Goossens, J. Phys. Chem. B 104, 7171 (2000).

${ }^{10}$ Z. Xie, V. M. Burlakov, B. M. Henry, C. R. M. Grovenor, H. E. Assender, and G. A. D. Briggs, in Materials for Photovoltaics, edited by Michael Durstock, Daniel Friedman, Russell Gaudiana, and Angus Rockett, Mater. Res. Soc. Symp. Proc., Vol. 836 (MRS, Warrendale, PA, 2005), p. L2.4.

${ }^{11}$ B. M. Henry, K. R. Kirov, M. J. Carey, C. R. M. Grovenor, H. E. Assender, G. A. D. Briggs, G. R. Webster, P. L. Burn, H. Uyama, and Y. Tsukahara, in Photovoltaic Science, Applications and Technology, edited by R. Gottschalg (The Solar Energy So- ciety, Vol. C79, p. 112.

${ }^{12}$ J. Nelson, A. M. Eppler, and I. M. Ballard, J. Photochem. Photobiol., A 148, 25 (2002).

${ }^{13}$ N. Golego, S. A. Studenikin, and M. Cocivera, Phys. Rev. B 61, 8262 (2000).

${ }^{14}$ S. A. Studenikin, N. Golego, and M. Cocivera, J. Appl. Phys. 84, 5001 (1998).

${ }^{15}$ N. Golego, S. A. Studenikin, and M. Cocivera, J. Mater. Res. 14, 698 (1999)

${ }^{16}$ S. A. Studenikin, N. Golego, and M. Cocivera, J. Appl. Phys. 83, 2104 (1998).

${ }^{17}$ A. Brajsa, K. Szaniawska, R. J. Barczynski, L. Murawski, B. Koscielska, A. Vomvas, and K. Pomoni, Opt. Mater. 26, 151 (2004).

${ }^{18}$ A. M. Eppler, I. M. Ballard, and J. Nelson, Physica E (Amsterdam) 14, 197 (2002).

${ }^{19}$ K. Pomoni, A. Vomvas, and C. Trapalis, Thin Solid Films 479, 160 (2005)

${ }^{20}$ B. Dulieu, J. Bullot, J. Wery, M. Richard, and L. Brohan, Phys. Rev. B 53, 10641 (1996).

${ }^{21}$ The situation is characteristic for photodoping, when the semiconductor can be considered as a doped one with light-intensity/ time dependent concentration of dopants.

${ }^{22}$ It can be shown that an increase in electrostatic energy associated with compensation of the trap charge by trapped hole charge is the same as if the trap were neutral and one considered the energy of electrostatic interactions between trapped holes.

${ }^{23}$ R. J. Gonzalez, R. Zallen, and H. Berger, Phys. Rev. B 55, 7014 (1997).

${ }^{24}$ C. Bauer, G. Boschloo, E. Mukhtar, and A. Hagfeldt, Chem. Phys. Lett. 387, 176 (2004). 